\title{
Vicenç Navarro, La situacion social en España
}

\section{Gregorio Rodriguez Cabrero}

\section{OpenEdition}

Journals

Édition électronique

URL : http://journals.openedition.org/travailemploi/4310

DOI : 10.4000/travailemploi.4310

ISSN : $1775-416 \mathrm{X}$

\section{Éditeur}

DARES - Ministère du Travail

\section{Édition imprimée}

Date de publication : 15 septembre 2008

Pagination : 112-113

ISSN : 0224-4365

\section{Référence électronique}

Gregorio Rodriguez Cabrero, «Vicenç Navarro, La situacion social en España », Travail et Emploi [En

ligne], 115 | juillet-septembre 2008, mis en ligne le 18 février 2011, consulté le 22 septembre 2020. URL : http://journals.openedition.org/travailemploi/4310 ; DOI : https://doi.org/10.4000/travailemploi. 4310 
ont un impact sur la dynamique salariale. Si tel était le cas, des inégalités salariales pourraient apparaître et décourager les travailleurs. À ce sujet, les résultats exposés par les auteurs montrent que le fait de connaître des périodes sans emploi provoque des pertes de salaire (importantes aussi bien en cas d'inactivité professionnelle qu'en cas de chômage). Cependant, dans certains pays, tels que la France et le Royaume-Uni, les effets néfastes du chômage sur les salaires ne sont que temporaires, contrairement à l'Espagne et à l'Italie où ceux-ci revêtent un caractère permanent. En cas d'interruption professionnelle volontaire, les conséquences s'avèrent positives lorsque la personne retrouve un emploi, ce qui n'est pas le cas en cas d'interruption professionnelle involontaire.

Parmi les autres facteurs à prendre en compte dans l'analyse des conséquences sur les salaires, on note la durée de la période de chômage ou le caractère volontaire ou involontaire de l'interruption professionnelle.

La formation au sein des entreprises constitue un autre sujet d'intérêt actuel relatif au marché du travail. Albert et al. concluent que ce sont les caractéristiques propres aux individus, au poste de travail et à l'entreprise - comme le niveau de formation, la taille de l'entreprise ou la branche d'activité du poste de travail - , et non celles relatives à l'ancienneté du travailleur, qui constituent les principaux facteurs déterminant l'accès à une formation. Par ailleurs, les auteurs estiment une équation de salaires avec effets fixes et concluent que la formation n'a pas d'impact significatif sur la croissance des salaires, ce qui signifie soit que la formation influe sur le niveau et non sur la croissance des salaires soit que la formation n'est pas proposée aux travailleurs appropriés.

Enfin, les conséquences des mutations sectorielles sont également à prendre en compte, tant au niveau des acteurs individuels qu'au niveau des institutions européennes du marché du travail. Parmi elles, l'importance croissante du secteur des services au sein des économies modernes joue un rôle prépondérant à l'heure actuelle. C'est pourquoi, Iglesias et Llorente analysent, en considérant que la mobilité professionnelle est exogène, les conséquences des mutations structurelles sur les marchés du travail. Ils montrent que l'industrie et le bâtiment connaissent les départs les plus importants alors que les embauches sont les plus fortes dans le secteur tertiaire qui, en plus d'être le secteur le plus porteur en termes d'offres d'emploi, est également créateur d'emplois dans les autres secteurs d'activité. De même, les auteurs concluent que les départs les plus nombreux concernent les emplois manuels alors que la majeure partie des embauches concerne les emplois non manuels. De plus, les emplois susceptibles de connaître le plus grand nombre de départs et d'embauches sont les emplois manuels hautement qualifiés.

Cet ouvrage offre donc une analyse complète de certaines questions essentielles relatives aux marchés du travail en Europe en proposant un large éventail d'informations visant à apporter un éclairage sur le degré d'intégration socio-économique, au niveau macro-économique, existant à l'heure actuelle au sein de l'Union européenne. Il conclut que nous sommes toujours au sein d'un processus de réforme des marchés du travail européens visant à parvenir à une plus grande cohésion. Les marchés du travail des différents pays membres présentent encore des disparités significatives en termes de fonctionnement. C'est pourquoi une intervention institutionnelle accrue, tant au niveau européen qu'à l'échelle nationale, s’impose afin d'aboutir à une plus grande flexibilité, de faciliter la conciliation entre vie familiale et vie professionnelle, de s'adapter aux processus de tertiarisation et de mondialisation propres aux marchés européens, d’accroître le degré de satisfaction professionnelle... et de parvenir à réduire les disparités existant entre les pays membres de l’Union européenne.

\section{La situacion social en España}

\author{
(La situation sociale en Espagne) \\ Vicenç Navarro (directeur)
}

Madrid: Biblioteca Nueva, 2007

\section{Lu par Gregorio Rodríguez Cabrero (universidad de Alcalá de Henares)}

L'ouvrage coordonné par le professeur Vicenç Navarro, de l'université Pompeu Fabra de Barcelone, constitue le second volume d'un dictionnaire commencé en 2006 qui vise à évaluer le développement et l'impact social des politiques sociales en Espagne. Ce dictionnaire fait partie intégrante d'un programme de politiques publiques et sociales de l'université mentionnée ci-dessus et de la Fondation F. Largo Caballero et constitue l'élément fondamental de l'Observatoire social espagnol (Observatorio Social de España). Cet observatoire ne produit pas seulement des dictionnaires, mais il met aussi à jour un site web qui abrite une banque de données sur les différents composants de l'Étatprovidence en Espagne.

Dans le cadre de cet ouvrage, qui poursuit le travail initié en 2006, quatorze chapitres consacrés à la recherche en sciences sociales se proposent d'aborder la réalité de la politique sociale espagnole. Les deux premiers chapitres, signés du professeur Navarro lui-même et de Jillian Reinolds, analysent les déficits de base de l'État-providence ainsi que la position relative que l'Espagne occupait en termes de protection sociale au sein des quinze pays que comptait l'Union européenne avant son extension à vingt-cinq. Ils analysent la subordination des dépenses publiques aux objectifs de l'union 
monétaire de sorte que le contrôle réussi du déficit et de la dette publique, en d'autres termes le succès de la convergence monétaire, contraste avec le déficit des dépenses publiques sociales ou déconvergence sociale. Dans la même optique d'analyse des dépenses publiques, ils mettent en avant le faible développement en Espagne de l'administration locale (Nuria Bosch et Marta Espasa), son peu d'implication dans les politiques sociales, état de fait que partagent les pays du sud de l'Europe et qui contraste avec la forte croissance de la responsabilité municipale dans d'autres modèles d'État-providence, comme celui des pays nordiques.

L'analyse du marché du travail (Javier RamosDíaz) et la prévention des risques liés au travail (Marcelo Amable et Joan Benach) constituent deux chapitres centraux de ce volume dans lesquels la forte création d'emplois en Espagne vient contraster avec quoi ? (principalement de femmes et d'immigrants non ressortissants des pays communautaires), facteur ayant permis au cours des trois années écoulées de faire passer le taux de chômage en dessous de la barre des dix pour cent, toutefois avec un taux élevé d'emplois précaires (aux alentours de $30 \%$ du total de la population active), un niveau élevé d'accidents du travail et des inégalités de condition de santé. Un ouvrage comme celui-ci ne pouvait pas ignorer le phénomène de l'immigration en Espagne et les politiques de contrôle des flux, de marché du travail et d'intégration sociale (Antonio Izquierdo). Le débat sur les processus de régularisation fait le cœur de ce chapitre et donne des éléments de réflexion pour les analystes européens.

L'analyse du système de santé espagnol de type universel de qualité relative, proposée par le professeur Navarro est abordée sous l'angle institutionnel. Elle concerne plus particulièrement la tendance récente de la transposition du système national de santé en dix-sept modèles sanitaires, autant qu'il y a de gouvernements régionaux, ainsi que la relative subordination des soins primaires à l' "hospitalocentrisme», avec ses inévitables listes d'attente et le blocage des soins spécialisés. Dans le même esprit, Maica Rodríguez, José Miguel Martínez, Gloria Pérez, María Buxó, Paloma Carrillo-Santisteve, Montse Vergara, Joan Benach et Carme Borrell analysent les inégalités de santé par catégorie sociale, par niveau de formation, genre et région.

La politique éducative est présentée aux chapitres 8, 9 et 10 du point de vue des inégalités sociales (Jorge Calero et Joseph-Oriol Escardíbul), du taux élevé d'échec scolaire, du rôle du corps enseignant formation, recrutement, conditions de travail et rétribution (Francesc Pedrós) et du déficit en nombre de jardins d'enfants (de 0 à 3 ans) (Pau Baizán et María José González), autant de facteurs propres à limiter l'accès des femmes ayant des enfants au marché du travail.

L'approbation de la loi sur l'autonomie personnelle et le soin aux personnes en situation de dépendance à la fin de l'année 2006 constitue un prétexte pour analyser (Sebastiá Sarasa) le faible niveau de couverture qu'offrent les services sociaux existants et les inégalités territoriales dans ce domaine. L'étude réalisée fait nettement apparaître que ces deux déficits entravent de manière extraordinaire l'élargissement de la nouvelle politique de protection sociale à la dépendance.

De même, l'ouvrage analyse également les difficultés structurelles d'accès au logement que connaissent les jeunes et les personnes sans foyer en Espagne dans le contexte d'une économie qui, au cours de la période de 1998 à 2007, s'est fondée sur l'industrie de la construction et dont l'un des effets collatéraux s'est traduit par de très fortes augmentations du prix du logement.

Le livre se termine, à l'instar du volume de 2006, sur l'analyse de l'impôt sur le revenu et ses effets redistributifs régressifs, personnels et territoriaux, comme conséquence des réformes fiscales menées en 1998 par le gouvernement conservateur du Parti populaire.

En résumé, le lecteur trouvera dans ces travaux rigoureux de lecture facile un guide pratique alliant informations et analyse et lui permettant de comprendre la structure et la dynamique des politiques sociales en général et sectorielle en Espagne pour la période allant approximativement de 2000 à 2006.

\section{La crisis de la ciudadania laboral}

(La crise de la citoyenneté au travail)

Alonso L. E., 2007(1)

\section{Barcelone, Anthropos Editorial}

\section{Lu par Celia Mayer Duque}

Le présent ouvrage compose le troisième volet d'une trilogie consacrée à la réflexion théorique sur les transformations de la norme d'emploi salariale. Les deux premiers volets, Travail et Citoyenneté: études sur la crise de la société salariale (Trotta, 1999) et Travail et postmodernité, la précarité de l'emploi (Fondements, 2001) ont précédé la rédaction de cet ouvrage qui, dans son ensemble, prétend être un travail en construction permanente enquêtant sur la place du travail dans la société actuelle.

L'auteur, partant d'une définition large du travail pris comme une relation sociale dans le plus pur style bourdieusien, analyse les transformations au sein du travail et de la citoyenneté survenues au cours des trente dernières années en partant de l'interrelation

(1) Nous remercions la revue Cuadernos de Relaciones Laborales (Cahiers sur les relations sociales) pour son autorisation de reproduction du présent compte rendu. 